\title{
Supratentorial Parenchymal Ependymomas in Children: Experience with 14 Patients at a Single Institute
}

\author{
Luxin YIN', Chunde $\mathrm{LI}^{2}$, Shiqi $\mathrm{LUO}^{2}$, Zhenyu MA² \\ ${ }^{1}$ The Affiliated Hospital of Xu Zhou Medical College, Department of Neurosurgery, Xuzhou, Jiangsu Province, China \\ ${ }^{2}$ Capital Medical University, Beijing Tiantan Hospital, Department of Neurosurgery, Beijing, China
}

\section{ABSTRACT}

AIM: To study the clinical features, imaging findings, treatment methods and results, and prognosis of supratentorial parenchymal ependymomas in children.

MATERIAL and METHODS: Fourteen children with supratentorial parenchymal ependymomas were treated by the senior author at Tiantan Hospital between October 2004 and May 2010. The clinical manifestations, neuroimaging findings, treatment methods, and therapeutic results were retrospectively reviewed.

RESULTS: The study included 11 boys and three girls. The patients were aged from 1 to 14 years with an average age of 6.1 years. Gross total surgical removal (GTR) was achieved in 10 cases, and subtotal removal (STR) was achieved in four cases. There were six ependymomas, seven anaplastic ependymomas, and one subependymoma. The mean follow-up duration was 78.9 months (range, 4-122 months). During the follow-up period, 10 patients who underwent GTR (71.4\%; only 1 patient had a recurrent tumor during this time) had an excellent postoperative outcome (Karnofsky Performance Status $\geq 80$ ), while four patients who underwent STR (28.6\%) developed recurrent tumors and died.

CONCLUSION: The preoperative diagnosis of supratentorial parenchymal ependymomas is difficult. GTR can be achieved with a favorable long-term outcome using an improved microsurgical technique.

KEYWORDS: Supratentorial ependymomas, Radiotherapy, Pediatrics, Histologic grade

\section{INTRODUCTION}

$\mathrm{E}$ pendymomas comprise $8 \%$ to $10 \%$ of brain tumors in children younger than 20 years and are slightly more common in male patients. Ependymomas are usually infratentorial, and approximately one-third is supratentorial. Among supratentorial ependymomas, extraventricular tumors account for $50 \%$, are frequently located adjacent to the cortical surface, and usually present with symptoms of high intracranial pressure $(2,3,5,7,9,12,16,22)$. Although this is a relatively rare central nervous system neoplasm, there is considerable controversy regarding the general nature of the tumor and treatment options $(2,3,9,18,19)$. In this study, we present 14 children who underwent neurosurgical operations for supratentorial parenchymal ependymomas, which have rarely been described in the literature. The clinical manifestations, neuroimaging findings, treatment methods, and therapeutic results were retrospectively reviewed.

\section{MATERIAL and METHODS}

\section{Patient Population}

From October 2004 to May 2010, a total of 14 consecutive children with supratentorial parenchymal ependymomas (age $<16$ years) underwent operations by the senior author in the Department of Neurosurgery of Beijing Tiantan Hospital, Capital Medical University, China. For this paper, all diagnoses 
were reviewed at the Department of Neuropathology at Beijing Tiantan hospital using the 2007 World Health Organization (WHO) classification of central nervous system tumors.

Written informed consent for inclusion in the study was provided by each patient or the patient's legal representative. The study protocol was approved by the ethics committee of Beijing Tiantan Hospital.

\section{Radiological Examinations}

Magnetic resonance imaging (MRI) is the diagnostic test of choice for ependymomas; it clearly demonstrates the location of the lesion and its relationship to surrounding tissue. All patients were evaluated preoperatively with computed tomography (CT) and contrast-enhanced MRI. Postoperative contrast-enhanced MRI was also performed for further evaluation of the extent of resection (Figure 1A-F).

\section{Surgical Procedure}

All patients were initially treated for their supratentorial parenchymal ependymomas. During the follow-up period, all patients with recurrent tumors underwent a second operation. We did not recommend adjuvant therapy such as radiotherapy or chemotherapy if gross total surgical removal (GTR) had been achieved in the patients with low grade tumors (The WHO Grade I and Grade II ependymomas). The radiotherapy should only be given to patients with anaplastic ependymomas and tumors in locations where total excision is not possible $(9,16,19,21)$.

\section{Pathology}

Pathologically, ependymomas are classified according to the WHO grading system. These tumors are categorized into myxopapillary (WHO Grade I), ependymoma (WHO Grade II) and anaplastic ependymoma (WHO Grade III). The WHO Grade I tumors are benign and are thought of as a separate clinical and pathological entity (2). The histological criteria of the WHO Grade II ependymomas include infrequent or absent mitoses, occasional nonpalisading foci of necrosis and nodules with better cellularity. Anaplastic ependymomas include tumors with clearly defined ependymal differentiation showing perivascular pseudorosettes and increased cellularity, cytological atypia, and microvascular proliferation (7).

The WHO Grade I and Grade II ependymomas are considered as the low grade tumors while the anaplastic ones are classified as the high grade tumors in this study.
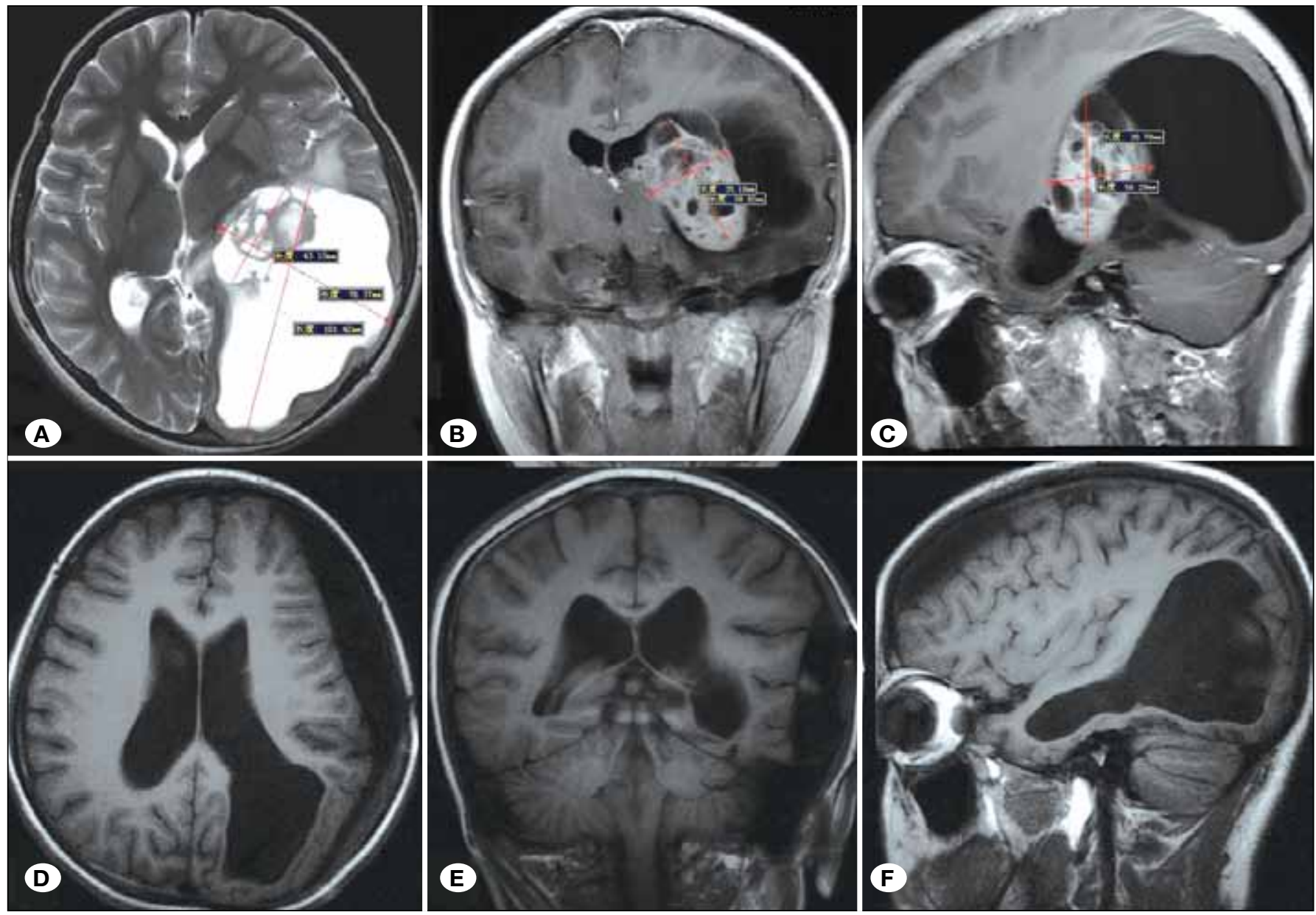

Figure 1: MRI showing a huge anaplastic ependymoma in a 14-year-old girl. A-C) Before the operation. D-F) After the operation. 


\section{Patient Follow-up}

Patients were followed up by clinical examination and contrastenhanced MRI at 2 and 6 months after surgery, and then every 1 or 2 years. Long-term follow-up data were collected from the outpatient files for most patients and by a telephone call or home visit for the other patients.

\section{- RESULTS}

\section{Clinical Data}

Fourteen patients were diagnosed with supratentorial parenchymal ependymomas, including 11 boys and three girls (male:female ratio, 3.67:1) with a mean age of 6.1 years (range, 1-14 years). The mean follow-up duration was 78.9 months (range, 4-122 months). The mean interval between the onset of symptoms and admission to our hospital was 2.2 months (range, 0.5-12 months). The clinical manifestations of supratentorial parenchymal ependymomas included signs of high intracranial pressure, including headache and vomiting in seven patients, seizure in three patients, and hemiparesis in five patients (Table I).

\section{Radiological Examination Findings}

Among all 14 patients, one lesion was located in the thalamus, two in the frontal lobe, two in the parietal lobe, one in the temporal lobe, and one in the frontotemporal lobe; moreover, three involved the junctional zone between the parietal lobe and temporal lobe, and four involved the junctional zone between the parietal lobe and frontal lobe (Table I).

Eleven patients had cystic ependymomas located at the supratentorium; the cystic regions had low signal intensity on $T_{1}$-weighted images $\left(T_{1} \mathrm{Wl}\right)$ and high signal intensity on $\mathrm{T}_{2} \mathrm{WI}$, and the solid regions had iso-intensity on both $\mathrm{T}_{1} \mathrm{WI}$ and $\mathrm{T}_{2}$ WI. Three patients had solid tumors with heterogeneous signal intensity on both $\mathrm{T}_{1} \mathrm{WI}$ and $\mathrm{T}_{2} \mathrm{Wl}$. After administration of contrast medium, the tumors demonstrated hyper-intensity with marked inhomogeneous enhancement.

\section{Surgical Procedures}

All patients initially underwent neurosurgical operations at our hospital. For tumors with huge cysts, puncture drainage was performed before surgical removal under MRI. GTR (>95\% resection by volume) was achieved in 10 patients, and subtotal removal (STR, $\leq 95 \%$ resection by volume) was achieved in four patients. Histological examination of the tumor demonstrated six ependymomas, seven anaplastic ependymomas, and one subependymoma. Five patients with recurrent tumors (one who underwent GTR and four who underwent STR at the first operation) underwent a second operation, and four of these five patients subsequently died (Table I).

\section{Pathology}

Seven patients had the WHO Grade III anaplastic ependymomas while 6 patients had the WHO Grade II ependymomas. Only one patient had the WHO Grade I subependymoma in our study.

\section{Patient Follow-up}

The 14 patients were followed up for a median period of 78.9 months (range, 4-122 months). During the follow-up period, 10 patients who underwent GTR (71.4\%; 5 anaplastic ependymomas, 4 ependymomas and 1 subependymoma, only 1 patient with ependymoma had a recurrent tumor during this time) had an excellent postoperative outcome (Karnofsky Performance Status, KPS $\geq 80$ ), while four patients who underwent STR $(28.6 \%, 2$ anaplastic ependymomas and 2 ependymomas) developed recurrent tumors and finally died.

\section{DISCUSSION}

Intracranial ependymomas are common brain tumors in children and comprise approximately $2 \%$ to $9 \%$ of all central nervous system tumors in this age category. Most intracranial ependymomas develop within the ventricular system, but they may arise from the extraventricular parenchyma as well (2$5,16)$. Supratentorial parenchymal ependymomas in children are a rarely seen pathological diagnosis. In this study, we reviewed 14 cases of supratentorial parenchymal ependymomas of children, and the clinical features, imaging findings, treatment methods and results, and prognosis of supratentorial parenchymal ependymomas were discussed.

\section{Clinical Data}

The presenting symptoms of supratentorial parenchymal ependymomas in children can be divided into symptoms caused by intracranial hypertension and those attributable to compression of neural structures (e.g., motor weakness and seizure) (3). The most important factors affecting the presenting symptoms are the tumor location and size (the medium diameter of the tumors in our series was $6.6 \mathrm{~cm}$ ).

The lesions in our series generally presented with focal neurological deficits (5 of 14 patients), seizures (3 of 14 patients), and high intracranial pressure secondary to a mass effect (7 of 14 patients). Symptoms of intracranial hypertension (e.g., headache, nausea, or vomiting) are the most common symptoms of pediatric supratentorial parenchymal ependymomas and are observed in most affected patients $(50 \%$ in our series). Headache is usually intermittent and may be worse in the morning. The mean duration from the onset of symptoms to the time of presentation is usually relatively short (2.2 months in our series) in relation to benign tumors.

\section{Neuroimaging}

Supratentorial parenchymal ependymomas are thought to originate from the remaining ependymal cells that have been retained within the brain parenchyma after embryonic development (20). These lesions tend to have cystic components (11 of 14 patients in our series) that are usually located near the trigone of the lateral ventricle. The solid parts of the tumor have equal or slightly lower intensity on $\mathrm{T}_{1} \mathrm{WI}$ and equally or slightly higher signal intensity on both $\mathrm{T}_{2} \mathrm{WI}$ and FLAIR $(1,6,8,9,17,20)$. Supratentorial parenchymal ependymomas have a higher tendency for calcification compared with infratentorial ependymomas. Although the MRI findings are somewhat characteristic, it is difficult to obtain a definite diagnosis before the operation. 


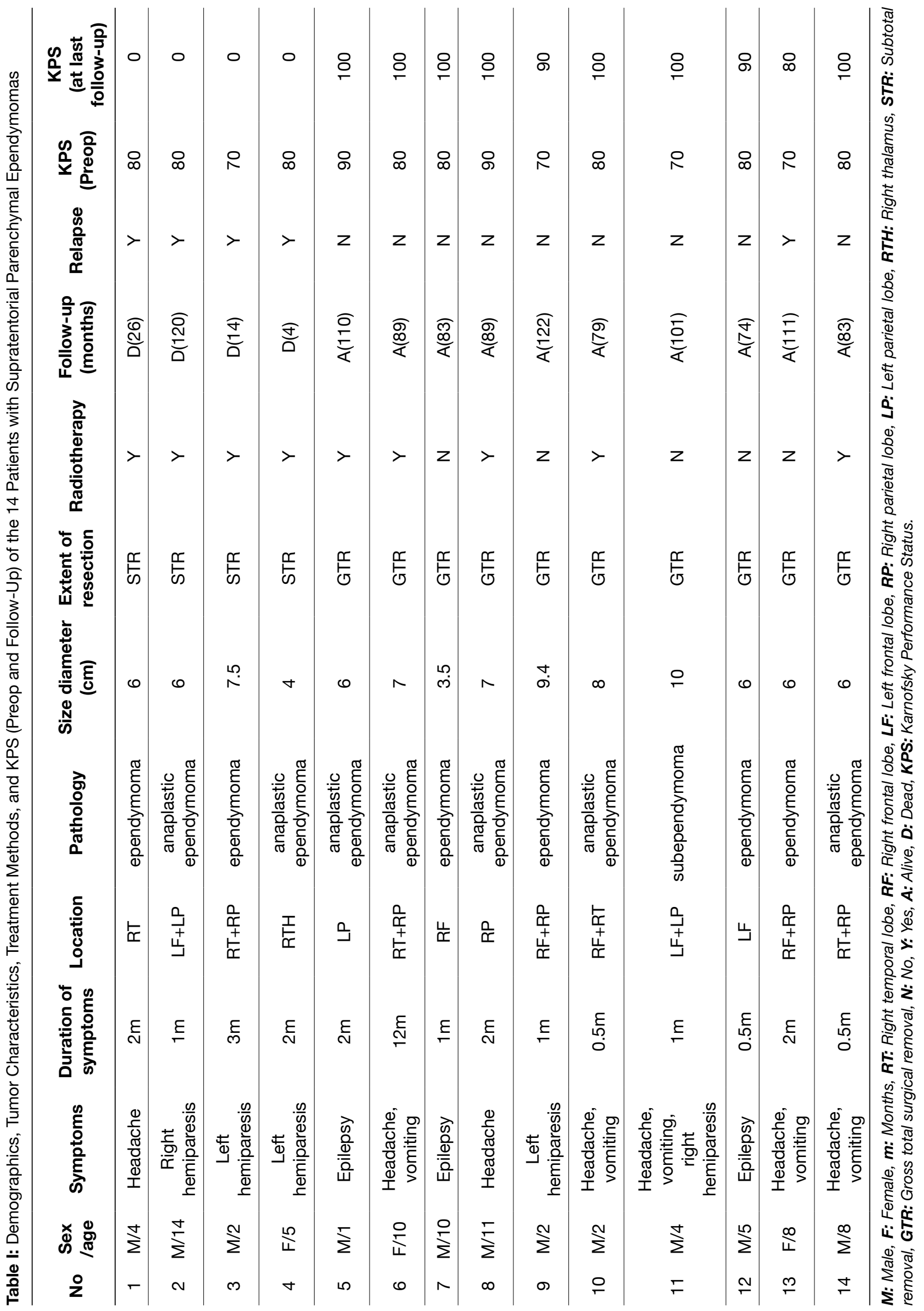


Yuh et al. thought that a thin rim of enhancement (likely representing granulation tissue) can be seen along the cavity margins beginning as soon as $24 \mathrm{~h}$ after surgery, and this rim becomes progressively thicker and more nodular over the next several days $(1,20)$ which progressively decreases beginning at approximately 5 weeks postoperatively and usually resolves completely within 12 months. Therefore, any new or increasing enhancement after approximately 5 weeks postoperatively should raise suspicion for tumor recurrence $(1,20)$. Thus, we recommend only CT scan in the next day after the operation to exclude hematoma and the contrast-enhanced MRI at 2 months after surgery to exclude recurrence if total removal of the tumor has been performed. Our results proved that our opinion was very appropriate for the treatment procedures.

\section{Treatment Procedures}

GTR (10 of 14 patients in our series) is the optimal therapy for symptomatic supratentorial parenchymal ependymomas $(2,3,5-9,12,15,18-21,23)$. In the present series, the postoperative care of patients depended on the operative procedure. Patients were sent to the neonatal intensive care unit after the operation. Head CT in the immediate postoperative period after craniotomy is reserved for patients who demonstrate delayed recovery from anesthesia or unexpected acute neurologic deficits (1).

Although the current therapy for ependymomas in children often includes surgery followed by radiotherapy, the current treatment recommendations for children vary and are often limited by the significant neurotoxicity associated with radiation. In general, it is considered safe to observe the patient when postoperative CT or MRI shows gross total excision, particularly when the tumor is of low grade $(3,9,19)$. Postoperative irradiation plays a role in the management of ependymomas, but indications for this adjuvant therapy remain a subject of debate.

Massimino et al. (10) presented 46 patients with GTR who were treated with hyperfractionated radiotherapy to a total dose of 70 Gy with $82 \% 5$-year progression-free survival rate. Jung et al. (4) described 30 patients in the past 15 years with a median dose of 52.8 Gy. The 5-year progression-free survival rate was $66.7 \%$. While there may be justification for adjunctive radiotherapy in patients with residual macroscopic disease, it has yet to be convincingly demonstrated that radiotherapy has additive benefit to surgery in children who have undergone complete resection. Little et al. (9) described four
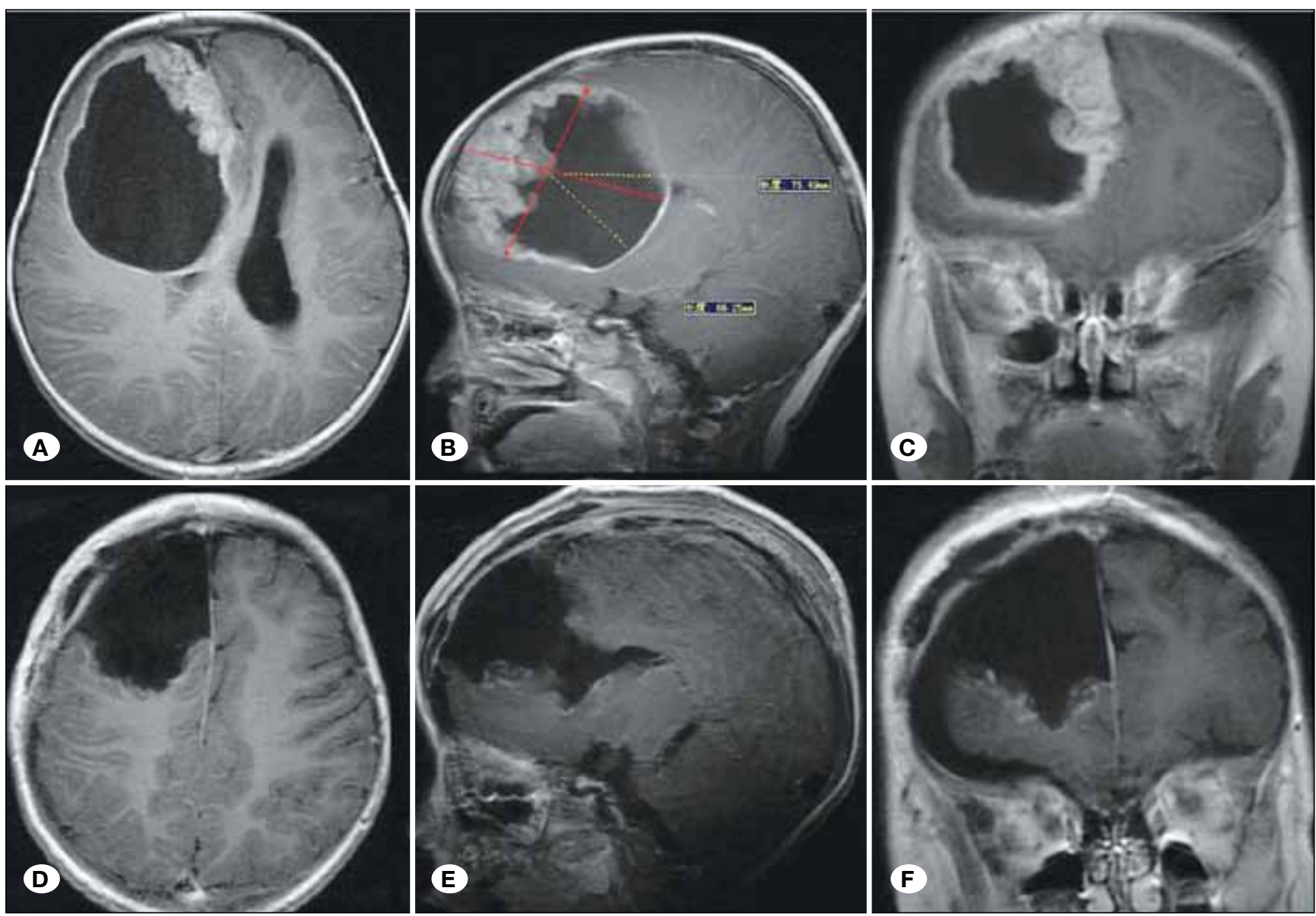

Figure 2: MRI showing a huge anaplastic ependymoma in a 2-year-old boy who had an excellent outcome. A-C) Before the operation. D-F) After the operation. 
patients with supratentorial ependymomas, including two with anaplastic ependymomas, who only underwent observation following complete resection, and all were alive at the last follow-up. Meanwhile, the results of the study by Palma et al. (14) of 6 patients did not demonstrate the significance of the radiotherapy as prognostic factor. Paulino et al. (15) also demonstrated no significant difference in prognosis in relation to radiotherapy which presented their experience with 49 patients.

In our series, it has been suggested that radiotherapy should only be given to patients with anaplastic ependymomas and tumors in locations where total excision is not possible (1012). The strategy for our therapy is appealing for several reasons. First, the radiotherapy was limited by the significant neurotoxicity associated with radiation. Second, the primary role of radiotherapy is to eradicate the microscopic residua in the operative bed responsible for tumor regrowth and dissemination (9). For the pediatric patients with low grade tumors (WHO I-II) who underwent GTR, the possibility of the tumor regrowth and dissemination is slim. Finally, we do not find any class I evidence which could demonstrate indications for this adjuvant therapy.

For the pediatric patients with high grade tumors (WHO III) or the ones who underwent STR, the radiotherapy was recommended at a radiation dose of 54 Gy at about 1 month after the initial surgery. Close observation with MRI should be performed in these patients. The success of this surgerybiased strategy depends on conscientious postoperative MRI follow-up and re-exploration if recurrence or progression is detected.

We favor close observation with MRI in patients with GTR. In our series, ten patients who underwent close observation after GTR, five of whom had anaplastic ependymomas (WHO III), had an excellent outcome with only one recurrence (Figure 2A-F). STR was only achieved for tumors located in critical locations or tightly attached to surrounding structures. For the patients whose tumors could be totally removed, after the initial surgery, if accessible residual tumor tissue is found on postoperative MRI, an early second-look surgery should also be proposed $(3,9,20)$.

\section{Prognosis}

Supratentorial parenchymal ependymomas represent a subgroup of tumors with a comparatively better prognosis $(71.4 \%$ of the patients had an excellent outcome postoperatively) than that of infratentorial ependymomas $(2,3,5,7,9,13,15)$. This may be related to the fact that total resection is more commonly achieved in patients with supratentorial tumors. Followup was achieved in all 14 patients with a median period of 78.9 months. Only one patient who received GTR underwent a second operation for the recurrent tumor, while all four patients who underwent STR subsequently died of recurrence (two patients received the second operation while the other two decline the operation). The patients who underwent GTR had a significantly more favorable prognosis (all alive at the last follow-up) than did those who underwent STR.

\section{CONCLUSION}

The preoperative diagnosis of supratentorial parenchymal ependymomas is difficult. GTR can be achieved with a favorable long-term outcome using an improved microsurgical technique. The radiotherapy should be given to patients with anaplastic ependymomas and tumors in locations where total excision is not possible. An early second-look surgery should also be proposed to achieve total excision in selected patients with accessible residual tumor tissue detected on postoperative MRI.

\section{- REFERENCES}

1. Barkovich AJ: Pediatric neuroimaging. London: Lippincott, Williams and Wilkins, 2005

2. Cage TA, Clark AJ, Aranda D, Gupta N, Sun PP, Parsa AT, Auguste $\mathrm{Kl}$ : A systematic review of treatment outcomes in pediatric patients with intracranial ependymomas. J Neurosurg Pediatr 11:673-681, 2013

3. Hou Z, Wu Z, Zhang J, Zhang L, Tian R, Liu B, Wang Z: Clinical features and management of intracranial subependymomas in children. J Clin Neurosci 20:84-88, 2013

4. Jung J, Choi W, Ahn SD, Park JH, Kim SS, Kim YS, Yoon SM, Song SY, Lee SW, Kim JH, Choi EK: Postoperative radiotherapy for ependymoma. Radiat Oncol J 30(4):158-164, 2012

5. Kilday JP, Rahman R, Dyer S, Ridley L, Lowe J, Coyle B, Grundy R: Pediatric ependymoma: Biological perspectives. Mol Cancer Res 7(6):765-786, 2009

6. Kilday JP, Rahman R, Dyer S, Ridley L, Lowe J, Coyle B, Grundy R: Childhood intracranial ependymoma: Twenty-year experience from a single institution. Cancer 110:432-441, 2007

7. Kumar R, Singhal N, Jaiswal SK, Mahapatra AK: Recurrence in supratentorial anaplastic ependymoma. Pediatr Neurosurg 43:364-368, 2007

8. Lefton DR, Pinto RS, Martin SW: MRI features of intracranial and spinal ependymomas. Pediatr Neurosurg 28:97-105, 1998

9. Little AS, Sheean T, Manoharan R, Darbar A, Teo C: The management of completely resected childhood intracranial ependymoma: The argument for observation only. Childs Nerv Syst 25:281-284, 2009

10. Massimino M, Gandola L, Giangaspero F, Sandri A, Valagussa P, Perilongo G, Garre ML, Ricardi U, Forni M, Genitori L, Scarzello G, Spreafico F, Barra S, Mascarin M, Pollo B, Gardiman M, Cama A, Navarria P, Brisigotti M, Collini P, Balter R, Fidani P, Stefanelli M, Burnelli R, Potepan P, Podda M, Sotti G, Madon El: Hyperfractionated radiotherapy and chemotherapy for childhood ependymomas: Final results of the first prospective AIEOP. Int J Radiat Oncol Biol Phys 58:1336-1345, 2004

11. Massimino M, Giangaspero F, Garre ML, Genitori L, Perilongo G, Collini P, Riva D, Valentini L, Scarzello G, Poggi G, Spreafico F, Peretta P, Mascarin M, Modena P, Sozzi G, Bedini N, Biassoni V, Urgesi A, Balestrini MR, Finocchiaro G, Sandri A, Gandola L: Salvage treatment for childhood ependymomas after surgery only: Pitfalls of omitting"at once"adjuvant treatment. Int J Radiat Oncol Biol Phys 65:1440-1445, 2006 
13. McGuire CS, Sainani KL, Fisher PG: Both location and age predict survival in ependymoma: A SEER study. Pediatr Blood Cancer 52:65-69, 2009

12. Merchant TE, Zhu Z, Thompson SJ, Sontag MR, Heideman RL, Kun LE: Preliminary results from a phase II trial of conformal radiation therapy for pediatric patients with localized lowgrade astrocytoma and ependymomas. Int $\mathrm{J}$ Radiat Oncol Biol 52:325-332, 2002

14. Palma L, Celli P, Mariottini A, Zalaffi A, Schettini G: The importance of surgery in supratentorial ependymomas: Longterm survival in a series of 23 cases. Childs Nerv Syst 16:170175,2000

15. Paulino AC, Wen BC, Buatti JM, Hussey DH, Zhen WK, Mayr $\mathrm{NA}$, Menezes AH: Intracranial ependymomas, an analysis of prognostic factors and patterns of failure. Am $\mathrm{J}$ Clin Oncol 25:117-122, 2002

16. Rousseau P, Habrand JL, Sarrazin D, Kalifa C, TerrierLacombe MJ, Rekacewicz C, Rey A: Treatment of intracranial ependymomas of children: Review of a 15-year experience. Int J Radiat Oncol Biol Phys 28:381-386, 1993
17. Spoto GP, Press GA, Hesselink JR, Solomon M: Intracranial ependymoma and subependymoma: MR manifestations. AJR Am J Roentgenol 154:837-845, 1990

18. Wang HC, Hertzog JH, O'Donnell HD, Walter AW: Natural progression of an unresected supratentorial ependymoma. Pediatr Neurosurg 44:75-78, 2008

19. Yadav YR, Neha, Chandrakar SK: Pure cortical supratentorial extraventricular ependymoma. Neurol India 57:213-215, 2009

20. Yuh EL, Barkovich AJ, Gupta N: Imaging of ependymomas: MRI and CT. Childs Nerv Syst 25:1203-1213, 2009

21. Yurt A, Selcuki M, Erturk AR, Kupelioglu A: Large supratentorial cortical ependymoma in a child. Clin Med Res 8:25-27, 2010

22. Zacharoulis S, Ji L, Pollack IF, Duffner P, Geyer R, Grill J, Schild $\mathrm{S}$, Jaing $\mathrm{TH}$, Massimino M, Finlay J, Sposto R: Metastatic ependymoma: A multi-institutional retrospective analysis of prognostic factors. Pediatr Blood Cancer 50:231-235, 2008

23. Zacharoulis S, Ashley S, Moreno L, Gentet JC, Massimino M, Frappaz D: Treatment and outcome of children with relapsed ependymoma: A multi-institutional retrospective analysis. Childs Nerv Syst 26(7):905-911, 2010 